\title{
On the Radical of a Hecke-Kiselman Algebra
}

\author{
Jan Okniński ${ }^{1} \cdot$ Magdalena Wiertel $^{1}$
}

Received: 9 March 2020 / Accepted: 16 September 2020 / Published online: 21 October 2020

(C) The Author(s) 2020

\begin{abstract}
The Hecke-Kiselman algebra of a finite oriented graph $\Theta$ over a field $K$ is studied. If $\Theta$ is an oriented cycle, it is shown that the algebra is semiprime and its central localization is a finite direct product of matrix algebras over the field of rational functions $K(x)$. More generally, the radical is described in the case of PI-algebras, and it is shown that it comes from an explicitly described congruence on the underlying Hecke-Kiselman monoid. Moreover, the algebra modulo the radical is again a Hecke-Kiselman algebra and it is a finite module over its center.
\end{abstract}

Keywords Hecke-Kiselman algebra · Monoid · Simple graph · Reduced words · Algebra of matrix type $\cdot$ Noetherian algebra $\cdot$ PI algebra $\cdot$ Jacobson radical

Mathematics Subject Classification 2010 16S15 - 16S36 - 16P40 - 16N20 - 16R20 · $20 \mathrm{M} 05 \cdot 20 \mathrm{M} 25 \cdot 20 \mathrm{C} 08 \cdot 05 \mathrm{C} 25$

\section{Introduction}

Let $\Theta$ be a finite simple oriented graph with $n$ vertices $\{1, \ldots, n\}$. The Hecke-Kiselman monoid $H K_{\Theta}$ associated to $\Theta$, introduced by Ganyushkin and Mazorchuk in [7], is generated by elements $x_{1}, \ldots, x_{n}$ subject to the defining relations:

(i) $x_{i}=x_{i}^{2}$, for $1 \leqslant i \leqslant n$,

(ii) $x_{i} x_{j}=x_{j} x_{i}$ if the vertices $i, j$ are not connected in $\Theta$,

(iii) $\quad x_{i} x_{j} x_{i}=x_{j} x_{i} x_{j}=x_{i} x_{j}$, if $i, j$ are connected by an arrow $i \rightarrow j$ in $\Theta$.

Thus, $H K_{\Theta}$ is a natural homomorphic image of the corresponding Coxeter monoid, where relations (iii) are replaced by the braid relations $x_{i} x_{j} x_{i}=x_{j} x_{i} x_{j}$. Hence, information

Presented by: Kenneth Goodearl

Magdalena Wiertel

mwiertel@mimuw.edu.pl

Jan Okniński

okninski@mimuw.edu.pl

1 Institute of Mathematics, University of Warsaw, Banacha 2, 02-097 Warsaw, Poland 
on the structure and representations of $H K_{\Theta}$ in particular contributes to the understanding of representation theory of the latter. Several combinatorial properties of $H K_{\Theta}$ and their representations were studied in $[1,5,7,9,11]$. We continue the study in [13], where the structure of $H K_{\Theta}$, and of the associated algebra $K\left[H K_{\Theta}\right]$ over a field $K$, is investigated. The case where $\Theta$ is the oriented cycle $x_{1} \rightarrow x_{2} \rightarrow \cdots \rightarrow x_{n} \rightarrow x_{1}$, with $n \geqslant 3$, plays a crucial role. Our first main result shows that the associated Hecke-Kiselman algebra, denoted by $K\left[C_{n}\right]$, is semiprime. It is also Noetherian, as shown in [13]. Consequently, since $K\left[C_{n}\right]$ is an algebra of Gelfand-Kirillov dimension one [10], from [16] it follows that $K\left[C_{n}\right]$ is a finite module over its center. Moreover, its classical quotient ring can be completely described.

Theorem 1 Let $n \geqslant 3$. Then $K\left[C_{n}\right]$ is a semiprime Noetherian PI-algebra. Moreover, its classical quotient ring is isomorphic to $\prod_{i=0}^{n-2} M_{n_{i}}(K(x))$, where $n_{i}=\left(\begin{array}{c}n \\ i+1\end{array}\right)$, for $i=$ $0, \ldots, n-2$.

Recall that the classical quotient ring of a semiprime Goldie PI-algebra is its central localization, see [14], Theorem 1.7.34.

In particular, this result answers a question asked in [13]. Next, we apply it to derive a description of the Jacobson radical $\mathcal{J}\left(K\left[H K_{\Theta}\right]\right)$ of an arbitrary algebra $K\left[H K_{\Theta}\right]$, provided it satisfies a polynomial identity. The latter condition is equivalent to a simple condition expressed in terms of the graph $\Theta,[10]$. Namely, it is equivalent to saying that $\Theta$ does not contain two cyclic subgraphs (i.e. subgraphs which are oriented cycles) connected by an oriented path. We prove that the radical is the ideal determined by an explicitly described congruence $\rho$ on $H K_{\Theta}$, so that $K\left[H K_{\Theta}\right] / \mathcal{J}\left(K\left[H K_{\Theta}\right]\right) \cong K\left[H K_{\theta} / \rho\right]$ is again a Hecke-Kiselman algebra and it has a very transparent structure. For a congruence $\eta$ on a semigroup $S$, the kernel of the natural homomorphism $K[S] \longrightarrow K[S / \eta]$ will be denoted by $I(\eta)$. So $K[S / \eta] \cong K[S] / I(\eta)$.

Namely, let $\rho$ be the congruence on $H K_{\Theta}$ generated by all pairs $(x y, y x)$ such that there is an arrow $x \rightarrow y$ that is not contained in any cyclic subgraph of $\Theta$. Let $\Theta^{\prime}$ be the subgraph of $\Theta$ obtained by deleting all arrows $x \rightarrow y$ that are not contained in any cyclic subgraph of $\Theta$. Then $H K_{\Theta} \cong H K_{\Theta} / \rho$. (If there is no such a pair then we assume that $\rho$ is the trivial congruence.) Then, because of the assumption that $K\left[H K_{\Theta}\right]$ is a PI-algebra, the connected components of $\Theta^{\prime}$ are either singletons or cyclic subgraphs. Recall from [13] that this implies that $K\left[H K_{\Theta}\right]$ is a Noetherian algebra. Indeed, Noetherian algebras $K\left[H K_{\Theta}\right]$ are characterized by the condition: each of the connected components of the graph $\Theta$ either is acyclic or it is a cyclic graph of length $n$ for some $n \geqslant 3$. Our second main result reads as follows.

Theorem 2 Assume that $\Theta$ is a finite oriented graph such that $K\left[H K_{\Theta}\right]$ is a PI-algebra. Let $\Theta^{\prime}$ be the subgraph of $\Theta$ obtained by deleting all arrows $x \rightarrow y$ that are not contained in any cyclic subgraph of $\Theta$ and let $\rho$ be the congruence on $H K_{\Theta}$ defined above. Then

1. the Jacobson radical $\mathcal{J}\left(K\left[H K_{\Theta}\right]\right)$ of $K\left[H K_{\Theta}\right]$ is equal to the ideal $I(\rho)$ determined by $\rho$,

2. $K\left[H K_{\Theta}\right] / \mathcal{J}\left(K\left[H K_{\Theta}\right]\right) \cong K\left[H K_{\Theta^{\prime}}\right]$ and it is the tensor product of algebras $K\left[H K_{\Theta_{i}}\right]$ of the connected components $\Theta_{1}, \ldots, \Theta_{m}$ of $\Theta^{\prime}$, each being isomorphic to $K \oplus K$ or to the algebra $K\left[C_{j}\right]$, for some $j \geqslant 3$,

3. $K\left[H K_{\Theta^{\prime}}\right]$ is a finitely generated module over its center. 
Recall that the Jacobson radical of a finitely generated PI-algebra $R$ is nilpotent, see [15], Theorem 6.3.39. However, for $R=K\left[H K_{\Theta}\right]$ this can also be derived from our proof.

This result opens a perspective for developing representation theory of such monoids $H K_{\Theta}$, which was one of the motivations in [7].

\section{Some background}

A Gröbner basis for $C_{n}$ has been found in [11], by applying the diamond lemma, see [2]. Consequently, the elements of $C_{n}$ can be treated as words in the free monoid $F=$ $\left\langle x_{1}, \ldots, x_{n}\right\rangle$ that are reduced in terms of certain rewriting system in $F$. Let $|w|_{i}$ denote the degree of a word $w$ (treated as an element of $C_{n}$ ) in the generator $x_{i}$. If $i, j \in\{1, \ldots, n\}$ then $x_{i} \cdots x_{j}$ denotes the product of all consecutive generators from $x_{i}$ up to $x_{j}$ if $i<j$, or down to $x_{j}$, if $i>j$.

Theorem 3 Let $\Theta=C_{n}$ for some $n \geqslant 3$. Let $S$ be the system of reductions in $F$ consisting of all pairs of the form

(1) $\left(x_{i} x_{i}, x_{i}\right)$ for all $i \in\{1, \ldots, n\}$,

(2) $\left(x_{j} x_{i}, x_{i} x_{j}\right)$ for all $i, j \in\{1, \ldots, n\}$ such that $1<j-i<n-1$,

(3) $\left(x_{n}\left(x_{1} \cdots x_{i}\right) x_{j}, x_{j} x_{n}\left(x_{1} \cdots x_{i}\right)\right)$ for all $i, j \in\{1, \ldots, n\}$ such that $i+1<j<n-1$,

(4) $\left(x_{i} u x_{i}, x_{i} u\right)$ for all $i \in\{1, \ldots, n\}$ and $1 \neq u \in F$ such that $|u|_{i}=|u|_{i-1}=0$. Here, we write $i-1=n$ if $i=1$,

(5) $\left(x_{i} v x_{i}, v x_{i}\right)$ for all $i \in\{1, \ldots, n\}$ and $1 \neq v \in F$ such that $|v|_{i}=|v|_{i+1}=0$. Here we write $i+1=1$ if $i=n$.

Then the set $\{w-v \mid$ for $(w, v) \in S\}$ is a Gröbner basis of the algebra $K\left[C_{n}\right]$.

It follows that an element $w \in F$ is a reduced word if and only if $w$ has no factors that are leading terms of the reductions (1) - (5) listed above. This reduction system is compatible with the degree-lexicographical ordering on the free monoid $F$ defined by $x_{1}<x_{2}<\cdots<$ $x_{n}$. We will use this result from [11] several times without further comment.

Our approach heavily depends on the results of [13]. In particular, a very transparent description of the reduced forms of almost all elements of $C_{n}$ has been found in [13], Theorem 2.1. Namely, for $i=0,1, \ldots, n-2$, the set $\tilde{M}_{i}$ of reduced forms of elements of $C_{n}$ that have a factor of the form $x_{n} q_{i}=x_{n} x_{1} \cdots x_{i} x_{n-1} \cdots x_{i+1}$ can be described as follows

$$
\tilde{M}_{i}=\left\{a\left(x_{n} q_{i}\right)^{k} b \in C_{n}: a \in A_{i}, b \in B_{i}, k \geqslant 1\right\},
$$

where $A_{i}, B_{i}$ are certain well defined sets. Moreover, if $\tilde{M}=\bigcup_{i=0}^{n-2} \tilde{M}_{i}$ then the set $C_{n} \backslash \tilde{M}$ is finite and each $M_{i}=\tilde{M}_{i}^{0}$ ( $\tilde{M}_{i}$ with zero adjoined) is isomorphic to a semigroup of matrix type $\mathcal{M}^{0}\left(S_{i}, A_{i}, B_{i} ; P_{i}\right)$, where $S_{i}$ denotes the cyclic semigroup generated by $s_{i}=$ $x_{n} q_{i}, P_{i}$ is a matrix of size $B_{i} \times A_{i}$ with coefficients in $\left\langle x_{n} q_{i}\right\rangle \cup\{\theta\}$, where $\left\langle s_{i}\right\rangle$ is the monoid generated by $s_{i}$. Recall that, if $S$ is a semigroup, $A, B$ are nonempty sets and $P=\left(p_{b a}\right)$ is a $B \times A$ - matrix with entries in $S^{0}$, then the semigroup of matrix type $\mathcal{M}^{0}(S, A, B ; P)$ over $S$ is the set of all triples $(s, a, b)$, where $s \in S, a \in A, b \in B$, with the zero element $\theta$, with operation $(s, a, b)\left(s^{\prime}, a^{\prime}, b^{\prime}\right)=\left(s p_{b a^{\prime}} s^{\prime}, a, b^{\prime}\right)$ if $p_{b a^{\prime}} \in S$ and $\theta$ otherwise. So, $\mathcal{M}^{0}(S, A, B ; P)$ is an order in the completely 0 -simple semigroup $\mathcal{M}^{0}(G, A, B ; P)$ over a cyclic infinite group, in the sense of [6]. Moreover $\mathcal{M}^{0}(K[S], A, B ; P)$ denotes the corresponding algebra of matrix type. It is defined as the contracted semigroup algebra 
$K_{0}\left[\mathcal{M}^{0}(S, A, B ; P)\right]$ and (if $A, B$ are finite) it can be interpreted as the set of all $A \times B$ - matrices over $K[S]$ with operation $\alpha \beta=\alpha \circ P \circ \beta$, where $\circ$ stands for the standard matrix product. For basic results on semigroups and algebras of matrix type we refer to [12], Chapter 5.They play a fundamental role in representation theory of semigroup algebras.

It is shown in [13] that $\left|A_{i}\right|=\left|B_{i}\right|$ and $P_{i}$ is not a zero divisor in the matrix ring $M_{n_{i}}\left(K\left[s_{i}\right]\right)$. Therefore, $P_{i}$ is invertible as a matrix in $M_{n_{i}}\left(K\left(s_{i}\right)\right)$ and hence the algebra of matrix type $\mathcal{M}^{0}\left(K\left(s_{i}\right), A_{i}, B_{i} ; P_{i}\right) \cong M_{n_{i}}\left(K\left(s_{i}\right)\right)$; this isomorphism is accomplished via the map $x \mapsto x \circ P$. Moreover, the latter is a central localization of the prime algebra $K_{0}\left[M_{i}\right] \cong \mathcal{M}^{0}\left(K\left[S_{i}\right], A_{i}, B_{i} ; P_{i}\right)$, where $S_{i}$ is the cyclic semigroup generated by $s_{i}$.

Lemma $1 K_{0}\left[M_{i}\right]$ is a prime algebra. Moreover, it does not have nonzero finite dimensional ideals.

Proof The first assertion was proved in [13], Theorem 5.8. Suppose that $J$ is a nonzero ideal. Then there exist $v, w \in M_{i}$ such that $v J w \neq 0$. Hence, the matrix type structure of $K_{0}\left[M_{i}\right]$ implies easily that there exist $v^{\prime}, w^{\prime} \in M_{i}$ such that $0 \neq v^{\prime} v J w w^{\prime} \subseteq K\left[x_{n} q_{i}\right]$. Then, clearly, $J \cap K\left[x_{n} q_{i}\right]$ is infinite dimensional.

We start with calculating the size of the set $A_{i}$, for every $i=0, \ldots, n-2$ and $n \geqslant 3$.

Proposition 4 For any $i \in\{0, \ldots, n-2\}$ and $n \geqslant 3$ we have $\left|A_{i}\right|=\left(\begin{array}{c}n \\ i+1\end{array}\right)$.

Proof For $i=n-2$ the assertion follows from Lemma 2.5 in [13], so next we assume that $i \leqslant n-3$.

From the description of the set $A_{i}$ from Theorem 2.1 in [13] it is clear that every element $w$ of $A_{i}$ is exactly of one of the forms

1. $w=\left(x_{k_{s}} \cdots x_{s}\right)\left(x_{k_{s+1}} \cdots x_{s+1}\right) \cdots\left(x_{k_{i+1}} \cdots x_{i+1}\right)$ where $i+1 \geqslant s \geqslant 1, s+1<k_{s+1}<$ $\cdots<k_{i+1} \leqslant n-1$ and $s \geqslant k_{s}$; for $s=i+1$ we assume that $w=\left(x_{k_{i+1}} \cdots x_{i+1}\right)$ with $i+1 \geqslant k_{i+1}$;

2. $w=\left(x_{k_{s}} \cdots x_{s}\right)\left(x_{k_{s+1}} \cdots x_{s+1}\right) \cdots\left(x_{k_{i+1}} \cdots x_{i+1}\right)$ where $i+1 \geqslant s \geqslant 1, s<k_{s}<$ $\cdots<k_{i+1} \leqslant n-1$

3. $w=1$.

Choose $1 \leqslant s \leqslant i+1$ and $0 \leqslant i \leqslant n-3$. Then the elements $w$ from Case 1 . are in a bijection with strictly increasing sequences $\left(k_{s}, \ldots, k_{i+1}\right)$ of natural numbers such that $1 \leqslant k_{s} \leqslant s<s+2 \leqslant k_{s+1}<\cdots<k_{i+1} \leqslant n-1$. It is easy to see that there exist exactly $s\left(\begin{array}{c}n-s-2 \\ i-s+1\end{array}\right)$ sequences of the above form. Similarly, elements $w$ of the form as in Case 2. are in a bijection with strictly increasing sequences $\left(k_{s}, \ldots, k_{i+1}\right)$ of natural numbers such that $s+1 \leqslant k_{s}<\cdots<k_{i+1} \leqslant n-1$. There are exactly $\left(\begin{array}{c}n-s-1 \\ i-s+2\end{array}\right)$ such sequences.

It follows that

$$
\left|A_{i}\right|=1+\sum_{s=1}^{i+1}\left(\left(\begin{array}{c}
n-s-1 \\
i-s+2
\end{array}\right)+s\left(\begin{array}{c}
n-s-2 \\
i-s+1
\end{array}\right)\right) .
$$

Thus, it is enough to prove that $1+\sum_{s=1}^{i+1}\left(\left(\begin{array}{c}n-s-1 \\ i-s+2\end{array}\right)+s\left(\begin{array}{c}n-s-2 \\ i-s+1\end{array}\right)\right)=\left(\begin{array}{c}n \\ i+1\end{array}\right)$ for $n \geqslant 3$ and $0 \leqslant i \leqslant n-3$. 
Moreover, if $i=n-3$, then by a direct calculation we get that

$$
1+\sum_{s=1}^{n-2}\left(\left(\begin{array}{l}
n-s-1 \\
n-s-1
\end{array}\right)+s\left(\begin{array}{l}
n-s-2 \\
n-s-2
\end{array}\right)\right)=\left(\begin{array}{c}
n \\
n-2
\end{array}\right)
$$

as desired.

It is easy to check that

$$
1+\sum_{s=1}^{i+1}\left(\left(\begin{array}{c}
n-s-1 \\
i-s+2
\end{array}\right)+s\left(\begin{array}{c}
n-s-2 \\
i-s+1
\end{array}\right)\right)=\sum_{k=0}^{i+1}(i+2-k)\left(\begin{array}{c}
n-i-3+k \\
k
\end{array}\right)
$$

Indeed, substituting $k=i+1-s$ in the sum in the left hand side, we get that this sum is equal to

$$
\begin{array}{r}
1+\sum_{k=0}^{i}\left(\begin{array}{c}
n-i-2+k \\
k+1
\end{array}\right)+\sum_{k=0}^{i}(i+1-k)\left(\begin{array}{c}
n-i-3+k \\
k
\end{array}\right)= \\
=1+\sum_{k=1}^{i+1}\left(\begin{array}{c}
n-i-3+k \\
k
\end{array}\right)+\sum_{k=0}^{i}(i+1-k)\left(\begin{array}{c}
n-i-3+k \\
k
\end{array}\right)= \\
=\sum_{k=0}^{i+1}\left(\begin{array}{c}
n-i-3+k \\
k
\end{array}\right)+\sum_{k=0}^{i+1}(i+1-k)\left(\begin{array}{c}
n-i-3+k \\
k
\end{array}\right)= \\
=\sum_{k=0}^{i+1}(i+2-k)\left(\begin{array}{c}
n-i-3+k \\
k
\end{array}\right),
\end{array}
$$

as claimed.

We proceed by induction on $n$ to prove that

$$
\sum_{k=0}^{i+1}(i+2-k)\left(\begin{array}{c}
n-i-3+k \\
k
\end{array}\right)=\left(\begin{array}{c}
n \\
i+1
\end{array}\right)
$$

For $i=0$ and arbitrary $n \geqslant 3$ we have $1+\left(\begin{array}{c}n-2 \\ 1\end{array}\right)+\left(\begin{array}{c}n-3 \\ 0\end{array}\right)=\left(\begin{array}{c}n \\ 1\end{array}\right)$ and the assertion follows. If $n=3$, then we have $0 \leqslant i \leqslant 0$, so the proposition holds.

Assume now that the equality is true for some $n$ and every $i \leqslant n-3$. Consider the sum

$$
\sum_{k=0}^{i+1}(i+2-k)\left(\begin{array}{c}
(n+1)-i-3+k \\
k
\end{array}\right)
$$

for $n-2>i>0$. Using $\left(\begin{array}{c}m+1 \\ k\end{array}\right)=\left(\begin{array}{c}m \\ k\end{array}\right)+\left(\begin{array}{c}m \\ k-1\end{array}\right)$ if $k \geqslant 1$ and $\left(\begin{array}{c}m+1 \\ 0\end{array}\right)=\left(\begin{array}{c}m \\ 0\end{array}\right)$ we get

$$
\begin{aligned}
& \sum_{k=0}^{i+1}(i+2-k)\left(\begin{array}{c}
(n+1)-i-3+k \\
k
\end{array}\right)= \\
& =\sum_{k=0}^{i+1}(i+2-k)\left(\begin{array}{c}
n-i-3+k \\
k
\end{array}\right)+\sum_{k=1}^{i+1}(i+2-k)\left(\begin{array}{c}
n-i-3+k \\
k-1
\end{array}\right) .
\end{aligned}
$$


From the induction hypothesis it follows that the first sum is equal to $\left(\begin{array}{c}n \\ i+1\end{array}\right)$. Substituting $m=k-1$ and $j=i-1$ we get

$$
\sum_{k=1}^{i+1}(i+2-k)\left(\begin{array}{c}
n-i-3+k \\
k-1
\end{array}\right)=\sum_{m=0}^{j+1}(j+2-m)\left(\begin{array}{c}
n-j-3+m \\
m
\end{array}\right) .
$$

From the induction hypothesis it follows that the above sum is equal to $\left(\begin{array}{l}n \\ i\end{array}\right)$. Now, using $\left(\begin{array}{c}n \\ i+1\end{array}\right)+\left(\begin{array}{c}n \\ i\end{array}\right)=\left(\begin{array}{c}n+1 \\ i+1\end{array}\right)$ we get

$$
\sum_{k=0}^{i+1}(i+2-k)\left(\begin{array}{c}
(n+1)-i-3+k \\
k
\end{array}\right)=\left(\begin{array}{c}
n \\
i+1
\end{array}\right)+\left(\begin{array}{c}
n \\
i
\end{array}\right)=\left(\begin{array}{c}
n+1 \\
i+1
\end{array}\right)
$$

and the assertion follows.

\section{Main results}

We will identify, without further comments, elements of the monoid $C_{n}$ with words in free monoid $F$ that are reduced with respect to the system $S$ described in Theorem 3.

Our first main aim is to show that $K\left[C_{n}\right]$ is semiprime. To prove this, we strengthen some of the results from [13].

Consider the automorphism $\sigma$ of $C_{n}$ given by $\sigma\left(x_{i}\right)=x_{i+1}$ for $i=1, \ldots, n$, where we agree that $x_{n+1}=x_{1}$. The natural extension to an automorphism of $K\left[C_{n}\right]$ also will be denoted by $\sigma$. For basic properties of this automorphism we refer to Section 3 in [13].

We have an ideal chain in $C_{n}$

$$
\emptyset=I_{n-2} \subseteq I_{n-3} \subseteq I_{n-4} \subseteq \cdots \subseteq I_{0} \subseteq I_{-1}
$$

where $I_{i}=\left\{w \in C_{n}: C_{n} w C_{n} \cap\left\langle x_{n} q_{i}\right\rangle=\emptyset\right\}$ for $i=0, \ldots, n-2$, and

$$
I_{-1}=I_{0} \cup C_{n} x_{n} q_{0} C_{n} .
$$

In particular, using Corollary 3.17 in [13] we obtain that $\sigma\left(I_{k}\right)=I_{k}$ for $k=0, \ldots, n-3$. The key structural result obtained in [13] reads as follows.

\section{Proposition 1 Consider the ideal chain (2). Then}

1. for $i=0, \ldots, n-2$, the semigroups of matrix type $M_{i}=\mathcal{M}^{0}\left(S_{i}, A_{i}, B_{i} ; P_{i}\right)$, satisfy $M_{i} \subseteq I_{i-1} / I_{i}$

2. for $i=1, \ldots, n-2$, the sets $\left(I_{i-1} / I_{i}\right) \backslash M_{i}$ are finite;

3. $I_{-1} / I_{0}=M_{0}$;

4. $\tilde{M}_{n-2}=M_{n-2} \backslash\{\theta\}$ is an ideal in $C_{n}$;

5. $C_{n} / I_{-1}$ is a finite semigroup.

The following observation can be deduced from the results and methods of [13].

Lemma $2 M_{i}$ is a right ideal in $C_{n} / I_{i}$ for every $i=0,1, \ldots, n-2$.

Proof Let $a\left(x_{n} q_{i}\right)^{k} b \in \tilde{M}_{i}$ and take any generator $x_{r} \in C_{n}$. Assume that the element $a\left(x_{n} q_{i}\right)^{k} b x_{r}$ is not in $\tilde{M}_{i}$. We claim that then $a\left(x_{n} q_{i}\right)^{k} b x_{r} \in I_{i}$. Let $b^{\prime}$ be the reduced form of $b x_{r}$. If $b^{\prime}=x_{j} \bar{b}$ for some word $\bar{b}$, where $j \leqslant i+1$, then using reduction (4) from Theorem 3 we get that $a\left(x_{n} q_{i}\right)^{k} b^{\prime}$ can be reduced to $a\left(x_{n} q_{i}\right)^{k} \bar{b}$. Therefore we can assume that a prefix 
of $b^{\prime}$ is equal to $x_{j}$, for some $j>i+1$. If $j<n$, then it can be calculated that $a\left(x_{n} q_{i}\right)^{k} b x_{r}$ can be rewritten as a word with a factor of the form $x_{j-1} \cdots x_{i+2} x_{n} x_{1} \cdots x_{i+1} x_{n-1} \cdots x_{j}$ and this element is in $I_{i}$ by Lemma 3.8 in [13]. Let us now consider the case when $x_{n}$ is a prefix of $b^{\prime}$. As we assume that $a\left(x_{n} q_{i}\right)^{k} b^{\prime} \notin \tilde{M}_{i}$, this word can be rewritten in $C_{n}$ as an element without the factor $x_{n} q_{i}$. From Theorem 3 it is easy to see that to obtain a word without such a factor one has to use a reduction of type (5). Therefore $a\left(x_{n} q_{i}\right)^{k} b^{\prime}$ can be written as a word with a prefix of the form $a\left(x_{n} q_{i}\right)^{k} x_{n} v x_{j}$, where $\left|x_{n} v\right|_{j}=\left|x_{n} v\right|_{j+1}=0$. Moreover, for $j \leqslant i$ or $j=n-1$ the generator $x_{j+1}$ occurs in $x_{n} q_{i} x_{n}$ after $x_{j}$, thus the reduction of $x_{j}$ of type (5) is not possible in this case. Therefore $n-1>j \geqslant i+1$. It follows (see Lemma 2.3 in [13]) that such a prefix is of the form $a\left(x_{n} q_{i}\right)^{k} x_{n} x_{1} \cdots x_{j}$. Therefore this element has a factor $x_{n} x_{1} \cdots x_{i} x_{n-1} \cdots x_{i+1} x_{n} x_{1} \cdots x_{j}$ for some $n-1>j \geqslant i+1$. It can be checked (using the reductions from Theorem 3) that the latter word can be rewritten as an element with the factor $x_{n-1} \cdots x_{j+1} x_{n} x_{1} \cdots x_{j}$, which is in $I_{j-1} \subseteq I_{i}$, by Lemma 3.8 in [13]. The assertion follows.

The following lemma provides a crucial step in the proof of Theorem 1 . By $\mathcal{P}\left(K\left[C_{n}\right]\right)$ we denote the prime radical of $K\left[C_{n}\right]$.

Lemma 3 Assume that $J$ is a finite dimensional ideal of $K\left[C_{n}\right]$. Then $J=0$. In particular, the left annihilator $A=\left\{\alpha \in K\left[C_{n}\right]: \alpha K[M]=0\right\}$ of $K[M]$ in $K\left[C_{n}\right]$ is zero. Moreover, $K\left[C_{n}\right]$ is a semiprime algebra.

Proof Suppose that $J \neq 0$ is a finite dimensional ideal of $K\left[C_{n}\right]$. First, we claim that a nonzero element $\alpha \in J$ can be chosen so that for every $i=1, \ldots, n$ we have $w x_{i}=w$ for all $w \in \operatorname{supp}(\alpha)$ or $\alpha x_{i}=0$.

Let $0 \neq \alpha \in J$ be such that $|\operatorname{supp}(\alpha)|$ is minimal possible. Let $\operatorname{supp}(\alpha)=\left\{v_{1}, \ldots, v_{k}\right\}$. Since $J$ is finite dimensional, the set $Z$ consisting of all such $k$-tuples is finite.

Let $\mathcal{R}$ denote the Green's relation on the monoid $C_{n}$, see [3]. Consider the $\mathcal{R}$-order $\leq \mathcal{R}$ on $C_{n}$; in other words, we write $w \leq_{\mathcal{R}} v$ if $w C_{n} \subseteq v C_{n}$. Then define a relation $\preceq$ on $C_{n}^{k}$ by: $\left(u_{1}, \ldots, u_{k}\right) \preceq\left(w_{1}, \ldots, w_{k}\right)$ if $u_{i} \leq_{\mathcal{R}} w_{i}$ for every $i=1, \ldots, k$.

Now, by the choice of $\alpha$, for every $x \in C_{n}$ we have that either $\alpha x=0$ or $\operatorname{supp}(\alpha x)=$ $\left\{v_{1} x, \ldots, v_{k} x\right\}$ and in the latter case $\left(v_{1} x, \ldots, v_{k} x\right) \preceq\left(v_{1}, \ldots, v_{k}\right)$. Since the set $Z$ introduced above is finite, we may further choose an element $\alpha$ for which the $k$-tuple $\left(v_{1}, \ldots, v_{k}\right)$ is minimal possible with respect to $\preceq$. Then $v_{i} \mathcal{R} v_{i} x$ for every $i$. Since the monoid $C_{n}$ is $\mathcal{J}$ trivial by [4], Theorem 4.5.3, it follows that for every $j$ we either have $w x_{j}=w$ for every $w \in \operatorname{supp}(\alpha)$ or $\alpha x_{j}=0$, as claimed.

Next, assume that $\beta \in K\left[C_{n}\right]$ is a nonzero element such that $w x_{1}=w$ holds in $C_{n}$ for every $w \in \operatorname{supp}(\beta)$. Then $|w|_{1}>0$ for every such $w$. Write $w=w_{0} x_{1} w_{1}$, for some reduced words $w_{0}, w_{1}$ such that $\left|w_{1}\right|_{1}=0$. We claim that then $\left|w_{1}\right|_{n}=0$. Indeed, if $w_{1}=u x_{n} v$ with $|v|_{n}=0$, then $w x_{1}=w_{0} x_{1} u x_{n} v x_{1}$ and then the only possible reduction that allows to decrease the length of this word (needed in order to get $w x_{1}=w$ in $C_{n}$ ) is of the form $x_{1} z x_{1} \rightarrow z x_{1}$, where $z$ is a prefix of $u x_{n} v$ containing $u x_{n}$. But then we do not get $w x_{1}=w$ in $C_{n}$ because $x_{1}$ appears after the last occurrence of $x_{n}$ in the reduced form of $w x_{1}$ ), a contradiction. So $\left|w_{1}\right|_{n}=0$, as claimed.

Assume first that $|w|_{n}>0$. Write $w=s x_{n} t x_{1} w_{1}$, for some reduced words $s, t$ (so $\left.w_{0}=s x_{n} t\right)$ such that $|t|_{n}=0$. Then also $|t|_{1}=0$ because $w$ is reduced. Hence, either $w x_{n}=s x_{n} t x_{1} w_{1} x_{n}$ is a reduced word with $\left|w x_{n}\right|_{n} \geqslant 2$ (if $\left|t w_{1}\right|_{n-1}>0$ ) or $w x_{n}=w$ in $C_{n}$ and the reduced form of $w x_{n}=w$ does not end with generator $x_{n}$ (if $\left|t w_{1}\right|_{n-1}=0$ ). 
Next, consider the case when $|w|_{n}=0$. It is clear that in this case $w x_{n}$ is a reduced word, and $\left|w x_{n}\right|_{n}=1$. Together with the previous paragraph of the proof this implies that $w x_{n} \neq w^{\prime} x_{n}$ in $C_{n}$ for all $w, w^{\prime} \in \operatorname{supp}(\beta)$ with $w \neq w^{\prime}$.

We have proved that the hypotheses on $\beta$ imply that $\beta x_{n} \neq 0$.

Now, we apply this observation to the element $\alpha$. Because of the choice of $\alpha$, we get that if $\alpha x_{1}=\alpha$ then $\alpha x_{n}=\alpha$. Using the automorphism $\sigma$ (and noting that $\sigma(\alpha)$, as an element of the finite dimensional ideal $\sigma(J)$ of $K\left[C_{n}\right]$, inherits the hypotheses on $\alpha$ ) we get that $\sigma(\alpha) x_{1}=\sigma(\alpha)$, so that $\sigma(\alpha) x_{n}=\sigma(\alpha)$, by the above argument applied to $\sigma(\alpha)$ in place of $\alpha$. Thus, $\alpha x_{n-1}=\alpha$, by applying $\alpha^{-1}$. Repeating this argument several times, we then get $\alpha x_{j}=\alpha$ for every $j$. A similar argument shows that if $\alpha x_{k} \neq 0$ for some $k$, then $\alpha x_{j} \neq 0$ for every $j$. However, $\alpha=\alpha x_{n} x_{1} x_{2} \cdots x_{n-1} \in J \cap K\left[\tilde{M}_{n-2}\right]$, a finite dimensional ideal of $K\left[\tilde{M}_{n-2}\right]$, because $x_{n} x_{1} \cdots x_{n-1} \in \tilde{M}_{n-2} \subseteq M$ and $\tilde{M}_{n-2}$ is an ideal of $C_{n}$. Therefore, Lemma 1 implies that $\alpha=0$. This contradiction shows that we may assume that $\alpha x_{j}=0$ for every $j$.

Let $w \in \operatorname{supp}(\alpha)$ be maximal with respect to the order $\leq_{\mathcal{R}}$. If $x_{j}$ is the last letter of the (reduced form of the) word $w$ then $w=w x_{j}=w^{\prime} x_{j}$ in $C_{n}$, for some $w^{\prime} \in \operatorname{supp}(\alpha)$. This implies that $w \leq \mathcal{R} w^{\prime}$, so by the choice of $w$ we get $w=w^{\prime}$, a contradiction. Therefore $J=0$.

By Theorem 5.9 in [13], $\mathcal{P}\left(K\left[C_{n}\right]\right) \cap K[\tilde{M}]=0$ because $\tilde{M}=\bigcup_{i=0}^{n-2} \tilde{M}_{i}$ and every $K\left[M_{i}\right]$ is prime. So, we know that $A \cap K[\tilde{M}]=0$ and therefore $A$ and $\mathcal{P}\left(K\left[C_{n}\right]\right)$ are finite dimensional, because $C_{n} \backslash \tilde{M}$ is finite. Hence, the assertion follows.

We are now in a position to prove Theorem 1.

Proof In view of Lemma 3, from Theorem 5.9 in [13] we know that $K\left[C_{n}\right]$ is a Noetherian semiprime PI-algebra.

For any fixed $i=0, \ldots, n-2$, let $J_{i}$ be a maximal among all ideals of $K\left[C_{n}\right]$ intersecting $K\left[x_{n} q_{i}\right]$ trivially and such that $K\left[I_{i}\right] \subseteq J_{i}$. Then $J_{i}$ is a prime ideal. By Corollary 10.16 in [8], $G K \operatorname{dim}(R)=c l K \operatorname{dim}(R)$ (the Gelfand-Kirillov and the classical Krull dimensions) for every finitely generated Noetherian PI-algebra $R$. Since $G K \operatorname{dim}\left(K\left[C_{n}\right]\right)=1$, it follows that $J_{i}$ is a minimal prime ideal of $K\left[C_{n}\right]$. Clearly, the image $J_{i}^{\prime}$ of $J_{i}$ in $K\left[C_{n}\right] / K\left[I_{i}\right]$ is a prime ideal. $M_{i}$ is a right ideal in $C_{n} / I_{i}$ by Lemma 3 , and thus it is a two-sided ideal because $C_{n} / I_{i}$ is endowed with a natural involution which preserves $M_{i}$, by Corollary 3.12 and Lemma 3.18 in [13]. Since $K\left[M_{i}\right]$ is a prime algebra, it follows that the classical quotient rings of $K\left[M_{i}\right]$ and $K\left[C_{n}\right] / J_{i}$ are equal. Moreover, as explained in the introduction, the classical ring of quotients of $K\left[M_{i}\right]$ is naturally isomorphic to $M_{n_{i}}(K(x))$, where $n_{i}=\left|A_{i}\right|$ for $i=0, \ldots, n-2$. Therefore, $J=\bigcap_{i=0}^{n-2} J_{i}$ is a semiprime ideal of $K\left[C_{n}\right]$ such that $J \cap K[M]=0$ (by the definition of the ideals $J_{i}$ ). Since $C_{n} \backslash M$ is finite, $J$ is finite dimensional, whence $J=0$ by Lemma 3. We obtain that the quotient ring $Q$ of $K\left[C_{n}\right]$ satisfies $Q \cong \prod_{i=0}^{n-2} M_{n_{i}}(K(x)), i=0, \ldots, n-2$. In view of Proposition 4, this completes the proof.

Our second main result describes the radical of a Hecke-Kiselman algebra $K\left[H K_{\Theta}\right]$, as well as the algebra modulo the radical, in the case of PI-algebras. So, assume that $\Theta$ is a finite oriented graph such that $K\left[H K_{\Theta}\right]$ is a PI-algebra. This is equivalent to saying that $\Theta$ does not contain two cyclic subgraphs (i.e. subgraphs which are cycles) connected by an oriented path, [10]. Let $\rho$ be the congruence on $H K_{\Theta}$ generated by all pairs $(x y, y x)$ such that there is an arrow $x \rightarrow y$ that is not contained in any cyclic subgraph of $\Theta$. (If there is 
no such a pair then we assume that $\rho$ is the trivial congruence.) Let $\Theta^{\prime}$ be the subgraph of $\Theta$ obtained by deleting all arrows $x \rightarrow y$ that are not contained in any cyclic subgraph of $\Theta$. Then $H K_{\Theta^{\prime}} \cong H K_{\Theta} / \rho$. Then the connected components of $\Theta^{\prime}$ are either singletons or cyclic subgraphs.

Now, we are in a position to prove Theorem 2.

Proof Suppose that a vertex $x \in V(\Theta)$ is a source vertex. In other words, there is an arrow $x \rightarrow y$ for some $y \in V(\Theta)$ but there are no arrows of the form $z \rightarrow x$. For any $w \in H K_{\Theta}$ consider the element $\beta=(x y-y x) w(x y-y x) \in K\left[H K_{\Theta}\right]$. Since $x$ is a source vertex, we know that $x v x=x v$ in $H K_{\Theta}$ for every $v \in H K_{\Theta}$. Hence $x w x y=x w y, x w y x=$ $x w y$. Similarly, $x y w x y=x y w y$ and $x y w y x=x y w y$. Therefore $\beta=0$. It follows that $x y-x y \in \mathcal{P}\left(K\left[H K_{\Theta}\right]\right)$.

If $x$ is a sink, that is there is an arrow $z \rightarrow x$ for some $z \in V(\Theta)$ but there are no arrows of the form $x \rightarrow y$ in the graph $\Theta$, a symmetric argument shows that $x z-z x \in \mathcal{P}\left(K\left[H K_{\Theta}\right]\right)$ for all $z$ such that $z \rightarrow x$ in $\Theta$. Let $\rho_{1}$ be the congruence generated by all pairs $(x y, y x)$ such that $x$ or $y$ is either source or sink and there is an arrow $x \rightarrow y$ that is not contained in any cyclic subgraph of $\Theta$. Equivalently, we may consider the graph $\Gamma_{1}$ obtained by erasing in $\Theta$ all such arrows $x \rightarrow y$ and $z \rightarrow x$ as above. Then $K\left[H K_{\Gamma_{1}}\right] \cong K\left[H K_{\Theta}\right] / I\left(\rho_{1}\right)$. We have shown that $I\left(\rho_{1}\right) \subseteq \mathcal{P}\left(K\left[H K_{\Theta}\right]\right)$. Repeating this argument finitely many times we easily get that $I(\rho) \subseteq \mathcal{P}\left(K\left[H K_{\Theta}\right]\right)$ (and our argument shows that $I(\rho)$ is nilpotent, because $\Theta$ is finite).

Since we know that $\mathcal{J}\left(K\left[H K_{\Theta}\right]\right)=\mathcal{P}\left(K\left[H K_{\Theta}\right]\right)$, to prove the first assertion of the theorem it is now enough to check that $K\left[H K_{\Theta^{\prime}}\right]$ is semiprime. $H K_{\Theta^{\prime}}$ is the direct product of all $H K_{\Theta_{i}}$, where $\Theta_{i}, i=1, \ldots, m$, are the connected components of $\Theta^{\prime}$. From [10] we know that each $H K_{\Theta_{i}}$ is either a band with two elements (if $\Theta_{i}$ has only one vertex) or it is isomorphic to $C_{k}$ for some $k \geqslant 3$. In the former case $K\left[H K_{\Theta_{i}}\right] \cong K \oplus K$, in the latter $K\left[H K_{\Theta_{i}}\right]$ is a semiprime PI-algebra (by Theorem 1) of Gelfand-Kirillov dimension one [10], and hence it is a finitely generated module over its center [16]. It follows easily that $K\left[H K_{\Theta}\right]$ is a finitely generated module over its center.

Let $Q_{i}$ be the classical ring of quotients of $K\left[H K_{\Theta_{i}}\right]$. If $H K_{\Theta_{i}}=C_{m_{i}}$ for some $m_{i}$ then we know that $Q_{i}$ is a central localization of the form described in Theorem 1. Clearly, $H K_{\Theta^{\prime}}$ is the direct product $\prod_{i=1}^{m} H K_{\Theta_{i}}$. Then in the localization $Q=Q_{1} \otimes$ $\cdots \otimes Q_{m}$ of $K\left[H K_{\Theta^{\prime}}\right] \cong \bigotimes_{i=1}^{m} K\left[H K_{\Theta_{i}}\right]$ each of the factors is isomorphic to $K \oplus K$ or to $\prod_{j=0}^{m_{i}-2} M_{r_{j}}(K(x))$, where $r_{j}=\left(\begin{array}{c}m_{i} \\ j+1\end{array}\right)$. Therefore, the tensor product is semiprime. Hence $K\left[H K_{\Theta^{\prime}}\right]$ is semiprime, because $Q$ is its central localization. It is now clear that $K\left[H K_{\Theta^{\prime}}\right] \cong K\left[H K_{\Theta}\right] / \mathcal{P}\left(K\left[H K_{\Theta}\right]\right)$. The result follows.

Acknowledgements This work was supported by grant 2016/23/B/ST1/01045 of the National Science Centre (Poland).

Open Access This article is licensed under a Creative Commons Attribution 4.0 International License, which permits use, sharing, adaptation, distribution and reproduction in any medium or format, as long as you give appropriate credit to the original author(s) and the source, provide a link to the Creative Commons licence, and indicate if changes were made. The images or other third party material in this article are included in the article's Creative Commons licence, unless indicated otherwise in a credit line to the material. If material is not included in the article's Creative Commons licence and your intended use is not permitted by statutory regulation or exceeds the permitted use, you will need to obtain permission directly from the copyright holder. To view a copy of this licence, visit http://creativecommonshorg/licenses/by/4.0/. 


\section{References}

1. Aragona, R., Andrea, A.D.: Hecke-Kiselman monoids of small cardinality. Semigroup Forum 86, 32-40 (2013)

2. Bergman, G.M.: The diamond lemma for ring theory. Adv. Math. 29, 178-218 (1978)

3. Clifford, A.H., Preston, G.B.: The Algebraic Theory of Semigroups, vol. 1. Amer. Math. Soc., Providence (1964)

4. Denton, T.: Algebra, Excursions into Combinatorics at $\mathrm{q}=0, \mathrm{PhD}$ thesis University of California, Davis arXiv:1108.4379 (2011)

5. Forsberg, L.: Effective representations of Hecke-Kiselman monoids of type A. arXiv:1205.0676v4 (2012)

6. Fountain, J., Petrich, M.: Completely 0-simple semigroups of quotients. J Algebra 101, 365-402 (1986)

7. Ganyushkin, O., Mazorchuk, V.: On Kiselman quotients of 0-Hecke monoids. Int. Electron. J. Algebra 10, 174-191 (2011)

8. Krause, G.R., Lenagan, T.H.: Growth of Algebras and Gelfand-Kirillov Dimension Revised Ed., Amer. Math. Soc., Providence (2000)

9. Kudryavtseva, G., Mazorchuk, V.: On Kiselman's semigroup. Yokohama. Math. J. 55, 21-46 (2009)

10. Męcel, A., Okniński, J.: Growth alternative for Hecke-Kiselman monoids. Publicacions Matemàtiques 63, 219-240 (2019)

11. Męcel, A., Okniński, J.: Gröbner basis and the automaton property of Hecke-Kiselman algebras. Semigroup Forum 99, 447-464 (2019)

12. Okniński, J.: Semigroup Algebras Monographs and Textbooks in Pure and Applied Mathematics, 138, Marcel Dekker, Inc., New York (1991)

13. Okniński, J., Wiertel, M.: Combinatorics and structure of Hecke-Kiselman algebras, Communications in Contemporary Mathematics 22, 2050022 (2020)

14. Rowen, L.H.: Polynomial Identities in Ring Theory, Academic Press, New York (1980)

15. Rowen, L.H.: Ring Theory, 2, Academic Press, New York (1988)

16. Small, L.W., Stafford, J.T., Warfield, R.B.: Affine algebras of Gelfand-Kirillov dimension one are PI. Math. Proc. Cambridge Philos. Soc. 97, 407-414 (1985)

Publisher's Note Springer Nature remains neutral with regard to jurisdictional claims in published maps and institutional affiliations. 